\title{
CONTEÚDO DE MICRONUTRIENTES NA BIOMASSA DE Acacia mearnsii De Wild.
}

\section{Micronutrients Content in Biomass of Acacia mearnsii De Wild.}

\author{
Leonir Rodrigues Barichello ${ }^{1}$ \\ Mauro Valdir Schumacher ${ }^{2}$ \\ Marcos Vinicius Winckler Caldeira ${ }^{3}$
}

\section{Resumo}

O presente estudo foi realizado em março de 2001 em um povoamento de Acacia mearnsii com 8 anos de idade, localizado no município de Minas do Leão/RS. Depois de avaliadas as características dendrométricas, foram selecionadas e abatidas 21 árvores do povoamento, sendo quantificados a biomassa e os micronutrientes nos componentes folhas; galhos vivos, galhos mortos; casca do tronco, madeira do tronco e raízes. Foram coletadas 42 unidades amostrais de serapilheira para quantificação de biomassa e micronutrientes. Para estimar a biomassa dos diferentes componentes, foram obtidos os coeficientes da equação $\ln \mathrm{y}=\mathrm{b}_{0}+\mathrm{b}_{1}$ * $\ln \mathrm{d}$. A produção de biomassa total foi de 132,1 $\mathrm{Mg} \mathrm{ha}^{-1}$. Essa biomassa continha um total de 4,06; 0,20; 4,79; 2,97; 0,69 kg ha' ${ }^{-1}$ dos micronutrientes B, Cu, Fe, Mn, Zn, respectivamente e 54,6 Mg ha' ${ }^{-1}$ de carbono orgânico. A biomassa de serapilheira acumulada sobre o solo foi de $25,7 \mathrm{Mg} \mathrm{ha}^{-1}$, contendo um total de 0,$74 ; 0,33 ; 23,05$; 2,81; 0,99 kg ha- ${ }^{-1}$ dos micronutrientes B, Cu, Fe, Mn e Zn, respectivamente e 8,9 Mg ha-1 de carbono orgânico. Palavras-chave: Acacia mearnsii, Micronutrientes, Carbono orgânico, Biomassa.

\section{Abstract}

This study was carried out in March 2001 in a 8 years old settlement of Acacia mearnsii De Wild, from AGROSETA S.A, in Minas do Leão county. After the evaluation of the dendrometrics characteristics, 21 trees were selected and cut, its biomass and macronutrients in the leaves, alive and dead branches, bark wood, stem wood and roots were quantified. Forty two (42) sampling unities of litter were collected to quantify the biomass and the micronutrients. To estimate the different components of the biomass, the coefficients of the equation $\ln =\mathrm{b}_{0}+\mathrm{b}_{1} * \ln \mathrm{d}$ were obtained. The production of total biomass was of $132.1 \mathrm{Mg} \mathrm{ha}^{-1}$. This total biomass contained 4.06; $0.20 ; 4.79 ; 2.97 ; 0.69 \mathrm{~kg} \mathrm{ha}^{-1} \mathrm{~B}, \mathrm{Cu}, \mathrm{Fe}, \mathrm{Mn}, \mathrm{Zn}$, respectively and $54.6 \mathrm{Mg} \mathrm{ha}^{-1}$ of organic carbon. The litter biomass was $25.7 \mathrm{Mg} \mathrm{ha}^{-1}$, containing of $0.74 ; 0.33 ; 23.05 ; 2.81 ; 0.99 \mathrm{~kg} \mathrm{ha}^{-1}$ of $\mathrm{B}, \mathrm{Cu}, \mathrm{Fe}$, $\mathrm{Mn}$ e $\mathrm{Zn}$, respectively and $8.9 \mathrm{Mg} \mathrm{ha}^{-1}$ of organic carbon.

Keywords: Acacia mearnsii, Nutrients, Organic carbon, Biomass.

Eng. Florestal, M. Sc. Cambará S/A. Rua Osvaldo Kroeff s/n, 95480-000, Cambará do Sul, RS. leonir.barichello@cambarasa.com.br Eng. Florestal, Dr. Prof. Adjunto do Departamento de Ciências Florestais/Universidade Federal de Santa Maria/RS. schuma@ufsm.br

3 Eng Florestal, Dr. Prof. do Departamento de Eng. Florestal/Centro de Ciências Tecnológicas/FURB - Universidade Regional de Blumenau. Rua São Paulo, 3250 - Campus II - Itoupava Seca - 89030-080, Blumenau/SC. caldeira@furb.br. Autor para correspondência. 


\section{Introdução}

A espécie florestal em estudo é originária do Sudeste da Austrália, encontrada, sobretudo, nas terras baixas, nas planícies costeiras e nos pequenos declives dos planaltos adjacentes perto de Sydney, New South Wales, Victoria, do Sul até o Sudeste da Austrália Meridional e Tasmânia (BOLAND et al., 1989). No Brasil, os plantios de Acácia-negra estão em altitudes que variam de 5 a 1.000 m s.n.m. (MANTOEFEL, 1991).

As acácias ocorrem e são cultivadas nas regiões tropicais e subtropicais da América do Sul, África do Sul, Ásia e Austrália, por outro lado não se encontram nas floras da Europa, Nova Zelândia e Antártica (BOLAND et al., 1989).

No sul do Brasil, existem diversas espécies de Acácias que são cultivadas para a produção de tanino, fins ornamentais e conservacionistas. Dentre essas, destacam-se Acacia mearnsii De Wild., Acacia decurrens (Wendl.) Wiled., Acacia dealbata Link., Acacia melanoxylon R. Br., Acacia podalyriifolia Cunn., Acacia longifolia (Andr.) Wiled. (MARCHIORI, 1990).

A matéria seca, conforme Larcher (1986), que constitui a biomassa, é formada especialmente por esqueletos de carbono e o restante por nutrientes minerais. As concentrações variam com a espécie, fase de desenvolvimento, estado nutricional, características edafoclimáticas e com a parte do vegetal.

Conforme Krapfenbauer citado por Shumacher (1995), na madeira das árvores, encontramse em média $50 \%$ de carbono, $4,3 \%$ de oxigênio, $6 \%$ de hidrogênio e $1 \%$ de elementos minerais. Segundo o mesmo autor, as acículas e folhas, a casca, componentes florais e sementes possuem teores maiores.

$\mathrm{O}$ acúmulo de nutrientes na biomassa arbórea varia de elemento para elemento em razão dos níveis de fertilidade do solo, das características nutricionais de cada espécie e da idade da floresta (SCHUMACHER, 1992; CALDEIRA, 2003).

A concentração de nutrientes nos tecidos vegetais reflete a influência da fertilidade do solo. De forma geral, os elementos de maiores concentrações são N, K e Ca, em torno de 10 a $50 \mathrm{~g} \mathrm{~kg}^{-1}$ da matéria seca, sendo que as folhas e os tecidos corticais acumulam a maior parte dos nutrientes. O lenho tem teores relativamente baixos, variando de 2 a $10 \mathrm{~g} \mathrm{~kg}^{-1}$ (LARCHER, 1986).
Na biomassa florestal, a distribuição de nutrientes varia ao longo do tempo. Por tanto, Freitas (2000) relata que, na fase juvenil, o processo é mais acelerado, estabilizando-se quando o povoamento atinge a maturidade.

De acordo com Haag (1985), na fase inicial do crescimento das plantas, a maior parte dos nutrientes está contida nas folhas. À medida que a idade aumenta, o peso seco e o teor de nutrientes variam, ocorrendo uma translocação dos nutrientes dos órgãos senescentes para as regiões de crescimento das árvores.

Os galhos, sobretudo os jovens, são importantes compartimentos de armazenamento de nutrientes (CALDEIRA, 1998; 2003). Os teores de nutrientes nos galhos novos são altos, sendo comparável ao das folhas, indicando uma reserva local de nutrientes a ser utilizada quando da expansão da copa, crescimento de galhos novos e folhas (DRIESSCHE citado por BELLOTE; SILVA, 2000).

Apesar da Acácia-negra ser plantada há mais de 50 anos no sul do Brasil, os estudos sobre teor de micronutrientes são raros. Nesse sentido, 0 presente estudo, envolvendo um povoamento de Acácia-negra com 8 anos de idade, teve como objetivos determinar o teor e o conteúdo de micronutrientes estocados na biomassa.

\section{Material e métodos}

\section{Caracterização do local de estudo}

O presente trabalho foi realizado em um povoamento de Acacia mearnsii pertencente à Empresa AGROSETA S/A, localizado no município de Minas do Leão, RS. O povoamento se localiza na região fisionômica natural do Estado do Rio Grande do Sul denominada Serra do Sudeste.

Segundo o sistema de classificação de Koeppen, o clima da região é do tipo Cfa, subtropical (MORENO, 1961). A temperatura média do mês mais quente (janeiro) é de $24^{\circ} \mathrm{C}$ e a do mês mais frio (julho) é de $13^{\circ} \mathrm{C}$ e a temperatura média anual é de $18-19^{\circ} \mathrm{C}$. A precipitação pluvial média nos meses de janeiro e julho e a precipitação anual são respectivamente $120-140$ mm, 120 mm e 1400 $\mathrm{mm}$.

De acordo com o Instituto de Pesquisas Agronômicas (1989), a região de Minas do Leão possui, de maio a agosto, 600 horas de frio abai- 
xo de $10^{\circ} \mathrm{C}$ e 200 horas de frio de maio a agosto abaixo de $7^{\circ} \mathrm{C}$. Em relação ao número de dias de chuvas, os meses de janeiro e julho têm, em média, 10 dias chuvosos. Anualmente, essa região tem, em média, 110 dias de chuva. A umidade relativa do ar, radiação solar e insolação anual são respectivamente $75-80 \%, 350 \mathrm{cal} \mathrm{cm}^{-2} \mathrm{dia}^{-1} \mathrm{e}$ 2400 horas.
Metodologia

Para a caracterização do solo na área experimental (QUADRO 1), foi aberto próximo a cada árvore amostrada uma trincheira de $60 \mathrm{~cm}$ de profundidade. Em cada uma das trincheiras, a cada 20 cm, fez-se uma coleta para determinação da densidade do solo, seguida de uma coleta de solo para análise edáfica do solo.

\section{Quadro 1 - Valores médios dos macro e micronutrientes obtidos na análise do solo, no local de plantio de Acacia mearnsii}

Chart 1 - Macro and micronutrients mean values from the soil analysis, in the site where the Acacia mearnsii was planted

\begin{tabular}{|c|c|c|c|c|c|}
\hline & & & Profu & ndidade $(\mathrm{cm}$ & \\
\hline & Unidade & $0-20$ & & $21-40$ & $41-60$ \\
\hline Densidade solo & g.cm ${ }^{-3}$ & & 1.42 & \begin{tabular}{l|l} 
& 1.44 \\
\end{tabular} & \begin{tabular}{l|l} 
& 1.39 \\
\end{tabular} \\
\hline Argila & & & 240 & 350 & 410 \\
\hline Matéria orgânica & g. $\mathrm{kg}^{-1}$ & & 23 & 21 & 14 \\
\hline $\mathrm{pH}\left(\mathrm{H}_{2} \mathrm{O}\right)$ & - & & 4.1 & $\overline{4.2}$ & 4.3 \\
\hline$\overline{\text { Nitrogênio total }}$ & & & 1.31 & 1.03 & 0.75 \\
\hline Fósforo assimilável & & & 8.5 & 3.9 & 2.2 \\
\hline Potássio trocável & g. $\mathrm{kg}^{-1}$ & & 120 & 137 & 91 \\
\hline Cálcio trocável & & & 0.9 & 0.6 & 0.4 \\
\hline Magnésio trocável & & & 0.4 & 0.3 & 0.3 \\
\hline Alumínio trocável & & & 2.2 & 3.6 & 4.3 \\
\hline Hidrogênio+Alumínio & & & 6.8 & 10.5 & 12.8 \\
\hline CTC $_{\text {efetiva }}$ & $-2-1$ & & 3.8 & 4.9 & 5.3 \\
\hline $\mathrm{CTC}_{\mathrm{pH} 7}$ & $\mathrm{CmOI}_{\mathrm{c}} \mathrm{L}^{-\mathrm{T}}$ & & 8.4 & 11.8 & 13.8 \\
\hline Saturação por bases (V) & & & 20 & 11 & 7 \\
\hline Saturação por alumínio (m) & $\%$ & & 57 & 73 & 82 \\
\hline Cobre & & & 1.4 & 1.5 & 1.6 \\
\hline Zinco & & & 0.8 & 0.7 & 0.3 \\
\hline Ferro & & & 346 & 356 & 227 \\
\hline Manganês & $\mathrm{mg} \cdot \mathrm{kg}^{-1}$ & & 11.6 & 4.4 & 2 \\
\hline Carbono orgânico & g.kg-1 & & 12.71 & 12.32 & 8.23 \\
\hline
\end{tabular}

A análise da densidade do solo foi realizada conforme o método do anel volumétrico de Kopecky (KIEHL, 1979). Os micronutrientes, bem como o carbono orgânico do solo foram determinados, segundo a metodologia proposta por Tedesco et al. (1995) e Miyazawa et al. (1999).

Os solos da região em estudo, conforme EMBRAPA (1973), pertencem à Unidade de Mapeamento São Jerônimo. De acordo com EMBRAPA (1999), o solo da região é caracterizado como Argissolo Vermelho Alítico típico, textura média/argilosa, relevo ondulado e substrato granito.
O trabalho foi realizado no mês de março de 2001, em um povoamento instalado em 1993, com densidade inicial de 1.960 plantas por ha $(3,0$ $\mathrm{m}$ entre linhas e 1,7 $\mathrm{m}$ entre plantas).

Inicialmente, em uma área com condições de sítio semelhante, foram demarcadas 7 parcelas de $18 \mathrm{~m} \mathrm{x} 24 \mathrm{~m}$, nas quais foi realizado o inventário florestal, medindo o DAP (diâmetro à altura do peito) de todas as árvores da parcela e altura de $20 \%$ delas. Na medição do DAP, foi utilizada fita métrica e na determinação da altura das árvores foi utilizado hipsômetro Vertex. Com base nos da- 
dos do inventário florestal, os diâmetros foram agrupados em classes, de maneira a abranger todas as variações do povoamento. O número de classes $(\mathrm{K})$ foi obtido mediante cálculos matemáticos pelo modelo: $K=1+3,3 * \log n$ em que: $n=$ número de elementos da amostra. E o intervalo de classes (h): $h=H / K$ em que: $H=$ amplitude total $\left(\mathrm{DAP}_{\text {máximo }}-\mathrm{DAP}_{\text {mínimo }}\right)$. Para estimar a altura das árvores que não foram medidas no campo foi utilizado o modelo de relação hipsométrica: $\mathrm{h}=\mathrm{b}_{0}+\mathrm{b}_{1} /$ $\mathrm{d}+\mathrm{b}_{2} / \mathrm{d}^{2}$ em que: $\mathrm{h}=$ altura estimada; $\mathrm{b}_{0}, \mathrm{~b}_{1}$ e $\mathrm{b}_{2}=$ coeficientes da equação; $d$ = diâmetro a altura do peito. Na determinação da biomassa acima do solo, foram abatidas 21 árvores no povoamento, sendo escolhidas três árvores dentro de cada classe de diâmetro anteriormente determinada $(5,1-8,0 ; 8,1$ 11,$0 ; 11,1-14,0 ; 14,1-17,0 ; 17,1-20,0 ; 20,1-23,0$ e 23,1$26,0)$, sendo a primeira com o DAP igual ao centro de classe, a segunda com o DAP 1,0 cm menor que o DAP do centro de classe e a terceira com 0 DAP 1,0 cm maior que o DAP do centro da classe. As 21 árvores abatidas tinham DAP de 5,5; 6,5; 7,5; 8,5; 9,5; 10,5; 11,5; 12,5; 13,5; 14,5; 15,5; 16,5; 17,5; 18,$5 ; 19,5 ; 20,5 ; 21,5 ; 22,5 ; 23,5 ; 24,5$ e $25,5 \mathrm{~cm}$.

Inicialmente, escolhia-se a árvore com 0 DAP desejado, logo após, esta foi derrubada rente ao solo, avaliando-se os seguintes componentes: folhas; galhos vivos, galhos mortos; casca do tronco e madeira do tronco. Imediatamente após o corte, foi coletada uma amostra de folhas do terço médio da copa nos quatro pontos cardeais para determinar o fator de umidade e para posterior análise química. Após isso, as árvores foram seccionadas e divididas em galhos, classificados em vivos (verdes) e mortos (secos), madeira e casca. Dos galhos vivos, foram separadas todas as folhas.

Todo o material morto foi pesado a campo com balança de gancho com precisão de $100 \mathrm{~g}$, e as amostras foram pesadas com balança digital com precisão de $1,0 \mathrm{~g}$. Logo, as amostras eram devidamente identificadas e enviadas para análises ao Laboratório de Ecologia Florestal do Departamento de Ciências Florestais da Universidade Federal de Santa Maria. No Laboratório as amostras foram armazenadas em estufa com circulação e renovação de ar a 75ํㅡ por um período de 72 horas, sendo resfriado em dessecadores e pesado em balança com $0,01 \mathrm{~g}$ de precisão. Posteriormente esse material foi moído em moinho tipo Wiley com mesch 30 .
As análises químicas dos micronutrientes foram determinadas segundo a metodologia proposta por Tedesco et al. (1995).

Na determinação da biomassa abaixo do solo, demarcou-se a área útil de cada planta $(1,7$ $\mathrm{m} \times 3,0 \mathrm{~m}=5,1 \mathrm{~m}^{2}$ ), escavando até $60 \mathrm{~cm}$ de profundidade, sendo separadas as raízes deste volume de solo, retirando uma amostra para determinação do fator de umidade e para posterior análise química. As raízes foram amostradas e pesadas a campo, sendo utilizada uma balança com precisão de 1,0 g para as amostras e as massas totais foram pesadas a campo com balança de gancho, possuindo uma precisão de $100 \mathrm{~g}$. Essas amostras também foram enviadas ao Laboratório de Ecologia Florestal para secagem, determinação de peso seco e moagem do material.

As coletas de serapilheira acumulada foram realizadas com o auxílio de uma moldura de ferro com dimensões de $25 \mathrm{~cm}$ x $25 \mathrm{~cm}$, em um número de 6 amostras por parcela, sendo 3 amostras na linha de cultivo e 3 amostras na entrelinha, totalizando 42 amostras na área estudada. Todo o material encontrado no interior do coletor foi devidamente recolhido, acondicionado em sacos de papel e levado ao Laboratório de Ecologia Florestal. No laboratório, o material foi posto para secar em estufa com circulação e renovação de ar, a uma temperatura de $75^{\circ} \mathrm{C}$ por um período de 72 horas. Após seco, o material foi resfriado em dessecadores e pesado em balança com $0,01 \mathrm{~g}$ de precisão. Logo após essas amostras foram enviadas ao Laboratório de Ecologia Florestal para secagem, determinação de peso seco e moagem do material.

\section{Resultados e discussão}

\section{Biomassa}

O Quadro 2 mostra que a produção de biomassa teve a seguinte ordem: madeira $>$ raízes $>$ galhos vivos $>$ casca $>$ galhos mortos $>$ folhas. Nesse povoamento, a biomassa do componente folha é menor que os demais componentes, pois é diferente de uma floresta em idade juvenil. Caldeira (1998), trabalhando em um povoamento de Acácia-negra, procedências Batemans Bay e Bodalla aos 2,4 anos de idade, encontrou a seguinte ordem: madeira $>$ folhas $>$ galhos vivos $>$ casca $>$ galhos mortos. 


\section{Quadro 2 - Biomassa média e percentual dos componentes das árvores de um povoamento de Acacia mearnsii}

Chart 2 - Biomass average and tree components percentage in a Acacia mearnsii stand

\begin{tabular}{|l|c|c|}
\hline Componente & Biomassa (Mg.ha- & Percentagem \\
\hline Folhas & 3,92 & 2,97 \\
Galhos mortos & 6,07 & 4,59 \\
Galhos vivos & 12,37 & 9,37 \\
Casca & 10,93 & 8,28 \\
Madeira & 82,35 & 62,34 \\
(total acima do solo & 115,64 & 87,55 \\
Raízes & 16,46 & 12,45 \\
Total & 132,10 & 100,00 \\
\hline
\end{tabular}

Fonte: Barichello et al. (2004)

A biomassa acima do solo representou $87,5 \%$ da biomassa total das árvores do povoamento, sendo que o fuste (madeira + casca) e a copa (folhas, galhos vivos e galhos mortos) representam 70,6 e 16,9\%, respectivamente, da biomassa total do povoamento.

\section{Micronutrientes}

Nas árvores de Acácia-negra, os maiores teores de micronutrientes foram encontrados nas folhas, enquanto que, na madeira, os menores (Quadro 3). A elevada concentração de macronutrientes nas folhas, em relação a outros componentes das árvores, torna este muito importante na ciclagem de nutrientes, embora apresentando um menor percentual em relação à biomassa total das árvores. Segundo Kozlowski; Pallardy (1996), nas folhas encontram-se a maioria das células vivas, tendendo a acumular maiores teores de macro e micronutrientes em razão dos processos de transpiração e fotossíntese.

Quadro 3 - Teor médio de micronutrientes nos componentes de biomassa de Acacia mearnsi Chart 3 - Mean micronutrients content of biomass components in Acacia mearnsii

\begin{tabular}{|l|c|c|c|c|r|}
\hline Componente & B & Cu & $\begin{array}{l}\text { Fe } \\
\mathbf{m g . k g}^{\mathbf{1}}\end{array}$ & Mn & Zn \\
\hline Folhas & 62,45 & 7,73 & 112,57 & 88,43 & 14,00 \\
Galhos mortos & 37,87 & 2,41 & 73,71 & 25,86 & 12,86 \\
Galhos vivos & 50,60 & 2,38 & 64,71 & 25,71 & 13,43 \\
Casca & 57,41 & 1,49 & 65,14 & 28,43 & 6,89 \\
Madeira & 24,94 & 0,92 & 24,71 & 13,79 & 3,46 \\
Raízes & 29,40 & 1,47 & 90,71 & 18,34 & 4,57 \\
\hline
\end{tabular}

Os teores de $\mathrm{Cu}, \mathrm{Fe}$, Mn e Zn do presente estúdio estão dentro das variações observadas por Bellote et al. (2002), em plantios de Acácia-negra aos 3 anos de idade.

Relacionando os teores de micronutrientes nas folhas com aqueles citados por Gonçalves; Valeri (2001) para Eucalyptus, os teores do B e do Cu estão acima da faixa adequada, os teores do Fe e Mn estão dentro da faixa de sufi- ciência e o teor de Zn está abaixo do considerado adequado.

O carbono orgânico variou entre 40,4\% e $43,3 \%$, sendo que o maior teor foi encontrado nas folhas (Quadro 4). De um modo geral, os componentes da copa (folhas e galhos) e casca decompõem-se mais rápido, enquanto o tronco e raízes têm uma taxa de decomposição mais lenta (Quadro 5). 


\section{Quadro 4 - Teor médio e conteúdo de carbono orgânico nos componentes de biomassa e de serapilheira de Acacia mearnsii}

Chart 4 -Mean proportion and content of organic carbon in biomass and litter components of Acacia mearnsii

\begin{tabular}{|l|c|c|}
\hline Componente & $\begin{array}{c}\text { Teor } \\
\mathbf{g . k g}^{\mathbf{1}}\end{array}$ & $\begin{array}{c}\text { Conteúdo } \\
\mathbf{k g . h}^{\mathbf{1}}\end{array}$ \\
\hline Folhas & 433,50 & $1.662,00$ \\
Galhos mortos & 410,10 & $2.483,05$ \\
Galhos vivos & 404,50 & $4.916,40$ \\
Casca & 407,00 & $4.448,19$ \\
Madeira & 416,90 & $34.365,56$ \\
Raízes & 416,40 & $6.736,41$ \\
\hline Total & - & $\mathbf{5 4 . 6 1 1 , 6 1}$ \\
\hline Serapilheira & 345,10 & $8.879,47$ \\
\hline
\end{tabular}

No ciclo de carbono, o dióxido de carbono $\left(\mathrm{CO}_{2}\right)$ fixado na biomassa é liberado para a atmosfera pelo processo de decomposição, sendo que a velocidade de liberação depende de fatores externos (temperatura e umidade) e da relação C/ $\mathrm{N}$ de cada componente da biomassa. Portanto, quanto menor for à relação $\mathrm{C} / \mathrm{N}$ mais rápida vai ser a liberação de $\mathrm{CO}_{2}$.

A rápida decomposição das folhas apresenta-se como uma excelente estratégia no processo de ciclagem dos nutrientes que auxiliam no crescimento dos diferentes componentes das árvores de Acácia-negra.

Quadro 5 - Relação entre C e N dos diferentes componentes da biomassa de Acacia mearnsii Chart 5 - Relation between $\mathrm{C}$ and Nin different biomass components of Acacia mearnsii

\begin{tabular}{|l|l|}
\hline Componente & Relação C/N \\
\hline Folhas & 14,39 \\
Galhos mortos & 52,04 \\
Galhos vivos & 47,87 \\
Casca & 39,44 \\
Madeira & 198,52 \\
Raízes & 95,07 \\
\hline
\end{tabular}

Conteúdo de micronutrientes

O conteúdo de micronutrientes em um povoamento florestal é conseqüência dos teores e da produção de biomassa nos diferentes compo- nentes das árvores (CALDEIRA, 2003). Nesse sentido, os maiores acúmulos de micronutrientes foram observados na madeira, exceto para o $\mathrm{Fe}$, que se apresentou um maior conteúdo nas raízes (Quadro 6). 
Quadro 6 - Conteúdo de micronutrientes nos componentes de biomassa de Acacia mearnsii Chart 6 - Micronutrients content of biomass components of Acacia mearnsii

\begin{tabular}{|l|l|l|l|l|}
\hline Componente & $\begin{array}{l}\text { B } \\
\text { kg.ha-1 }\end{array}$ & Cu & Fe & Mn Zn \\
\hline Folhas & 0,24 & 0,03 & 0,39 & $0,350,06$ \\
Galhos mortos & 0,23 & 0,02 & 0,43 & $0,180,07$ \\
Galhos vivos & 0,62 & 0,03 & 0,6 & $0,330,14$ \\
Casca & 0,54 & 0,02 & 0,65 & $0,350,07$ \\
Madeira & 1,95 & 0,08 & 0,92 & $1,40,27$ \\
Raízes & 0,49 & 0,03 & 1,79 & $0,350,08$ \\
Total & 4,07 & 0,21 & 4,78 & $2,960,69$ \\
\hline
\end{tabular}

Serapilheira

No povoamento de Acácia-negra com 8 anos de idade, a produção de serapilheira sobre a superfície do solo foi de aproximadamente 25,73 Mg ha-1.
O teor e o conteúdo de micronutrientes na serapilheira no povoamento de Acácia-negra podem ser observados na Quadro 7. A distribuição dos micronutrientes na serapilheira teve a seguinte ordem: $\mathrm{Fe}>\mathrm{Mn}>\mathrm{Zn}>\mathrm{B}>\mathrm{Cu}$. Esses micronutrientes somaram um total de $27,92 \mathrm{~kg} \mathrm{ha}^{-1}$.

\section{Quadro 7 - Teor e conteúdo de micronutrientes na serapilheira de um povoamento de Acacia mearnsii}

Chart 7 - Mean micronutrients content in litter of a Acacia mearnsii stand

\begin{tabular}{|l|l|l|l|l|l|}
\hline Teor & $\begin{array}{l}\text { B } \\
\text { mg.kg }\end{array}$ & Cu & Fe & Mn & Zn \\
\hline Conteúdo & 28,68 & 12,85 & 895,83 & 109,33 & 38,33 \\
& $\begin{array}{l}\mathrm{kg}^{\mathbf{1}} \mathrm{ha}^{-1} \\
0,74\end{array}$ & 0,33 & 23,05 & 2,81 & 0,99 \\
\hline
\end{tabular}

O elevado teor e conteúdo de Fe na serapilheira podem ser atribuídos à contaminação com partículas de solo. Tal observação também foi feita por Neves (2000), trabalhando com serapilheira de diferentes clones de Eucalyptus sp e por Caldeira (2003), trabalhando com a Floresta Ombrófila Mista Montana, General Carneiro/PR.

Calil (2003), trabalhando em um povoamento de Acácia-negra no município de Tupanciretã/RS, encontrou teores de Fe, Mn e Zn superiores ao observado no presente trabalho, por outro lado, os teores de $\mathrm{B}$ e $\mathrm{Cu}$ foram inferiores. Contudo, a ordem de distribuição entre eles manteve-se a mesma.

\section{Conclusões}

Os componentes "folhas das árvores de Acácia-negra" e "madeira" apresentaram os maiores e menores teores de micronutrientes analisados, respectivamente.

O conteúdo de micronutrientes na biomassa total teve a seguinte ordem decrescente: $\mathrm{Fe}>\mathrm{B}>\mathrm{Mn}>\mathrm{Zn}>\mathrm{Cu}$.

Na biomassa de serapilheira, o conteúdo de micronutrientes apresentou a seguinte ordem decrescente: $\mathrm{Fe}>\mathrm{Mn}>\mathrm{Zn}>\mathrm{B}>\mathrm{Cu}$. 


\section{Referências}

BARICHELLO, L R; SCHUMACHER, M. V.; CALDEIRA, M. V. W. Quantificação da biomassa em floresta de Acacia mearnsii De Wild. na região sul do Brasil. In $\therefore$ CONGRESSO INTERNACIONAL DE BIOENERGIA 1., 2004, Campo Grande. Anais... Viçosa: Renabio, 2004. (no prelo).

BELLOTE, A. F. J.; SILVA, H. D. Técnicas de amostragem e avaliações nutricionais em plantios de Eucalyptusspp. In . GONÇALVES, J. L. M.; BENEDETTI, V. Nutrição e fertilização florestal. Piracicaba: Instituto de Pesquisas e Estudos Florestais, 2000. p. 105-133.

BOLAND, D. J. et al. Forest trees of Australia. Melbourne, Australia: Thomas Nelson Australia/ CSIRO, 1989. p. 687.

CALDEIRA, M. V. W. Determinação de biomassa e nutrientes em uma Floresta Ombrófila Mista Montana em General Carneiro, Paraná. 2003. 176f. Tese (Doutorado em Ciências Florestais) Setor de Ciências Agrárias, Universidade Federal do Paraná, Curitiba, 2003.

CALDEIRA, M. V. W. Quantificação da biomassa e do conteúdo de nutrientes em diferentes precedências de Acácia-negra (Acacia mearsn sii De Wild.). 1998. 96f. Dissertação (Mestrado) Universidade Federal de Santa Maria, Santa Maria, 1998.

CALIL, F. N. Aspectos da ciclagem de nutrientes em um sistema silvopastoril na região de Tupanciretã. 2003. Santa Maria:UFSM, 2003. (Relatório técnico).

EMPRESA BRASILEIRA DE PESQUISA AGROPECUARIA. Levantamento de reconhecimento dos solos do Estado do Rio Grande do Sul. Recife, 1973. (Boletim Técnico, 30).

EMPRESA BRASILEIRA DE PESQ UISA AGROPECUARIA. SNPS Sistema brasileiro de classificação e levantamento de solos. Rio de Janeiro,RJ: EMBRAPA, 1999. p. 412.

FREITAS, R. A. Estudo de biomassa e do conteúdo de nutrientes em um povoamento de Eucalyptus grandis Hill ex Maiden plantado em solo sujeito a arenização no município de Alegrete-RS. 2000. 60f. Dissertação (Mestrado em
Engenharia Florestal) - Universidade Federal de Santa Maria, Santa Maria, 2000.

GONÇALVES, J. L. M.; VALERI, S. V. Eucalipto e Pinus. In FERREIRA, M.E.; CRUZ, M.C.P.; RAIJ, B.V.; ABREU, C.A. Micronutrientes e elementos tóxicos na agricultura. Jaboticabal: CNPq/FAPESP/POTAFOS, 2001. p. 393-423.

HAAG, H. P. Ciclagem de nutrientes em florestas tropicais. Campinas: Fundação Cargill, 1985. p. 114.

INSTITUTO DE PESQUISAS AGRONÔMICAS. Seção de Ecologia Agrícola. Atlas agroclimático do Estado do Rio Grande do Sul. Porto Alegre, 1989. v. 3.

KIEHL, E. J. Manual de edafologia. São Paulo,SP: Ceres, 1979. p. 2262.

KOZLOWSKI, T. T.; PALARDY, S. G. Physiological of woody. 2. ed. San Diego: Academic, 1996. p. 432.

LARCHER, W. Ecofisiologiavegetal. São Paulo,SP: EPU, 1986. p. 319.

MANTOEFEL, J. C. Reflorestamento no setor privado - Acacicultura. In: SEMINÁRIO SOBRE SITUAÇÃO FLORESTAL DO RIO RANDE DO SUL, 1.; 1991. Santa Maria. Anais... Santa Maria: UFSM/ CEPEF/FATEC, 1991. p. 108-114.

MARCHIORI, J. N. C. Anatomia das madeiras do gênero acácia, nativas e cultivadas no Estado do Rio Grande do Sul. 1990. 226f. Tese (Doutorado em Engenharia Florestal) - Universidade Federal do Paraná, Curitiba, 1990.

MIYAZAWA, M.; PAVAN, M.; MURAOKA, T.; CARMO , C. A. F. S.; MELLO, W. J. Análises químicas de tecido vegetal. In: SILVA, F. C. (ed). Manual de análises químicas de solos, plantas e fertilizantes. Brasília: Embrapa Comunicação para Transferência e Tecnologia, 1999. p. 171-223.

MORENO, J.A. Clima do Rio Grande do Sul. Porto Alegre: Secretaria da Agricultura, 1961. p. 42.

NEVES, J. C. L Produção e participação de biomassa, aspectos nutricionais e hídricos em plantios clonais de eucalipto na região litorânea do Espírito Santo. 2000. 191f. Tese (D outorado em Produção Vegetal) - Universidade Estadual do Norte Fluminense, Rio de Janeiro, 2000. 
SCHUMACHER, M. V. Aspectos da ciclagem de nutrientes e do microclima em talhões de Eucalyptus camaldulensis Dehnh., Eucalyptus grandisW. Hill ex Maiden e Eu calyptustorelliana F. Muell. 1992. 87f. Dissertação (Mestrado em Ciência Florestal) - Escola Superior de Agricultura "Luiz de Queiroz" - Universidade de São Paulo, Piracicaba, 1992.
SCHUMACHER, M.V. Naehrstoffkreislauf in verschiedenen Bestaenden von Eucalyptus saligna (Smith), Eucalyptusdunnii (Maiden) und Eucalyptus globulus (Labillardière) in Rio Grande do Sul, Brasilien. 1995. 167f. Tese (Doutorado) - Viena, Áustria, 1995.

TEDESCO, M J.; GIANELLO, C.; BISSANI, C. A.; BOHNEN, H.; VOLKWEISS, S. J. Análises de solos, plantas e outros materiais. 2. ed. Porto Alegre: UFRGS, 1995. (Boletim Técnico, 5).

Recebido: 17/09/2004

Aprovado: 31/01/2005 